\title{
Towards Benchmarking Feature Type Inference for AutoML Platforms
}

\author{
Vraj Shah \\ University of California, San Diego \\ vps002@eng.ucsd.edu
}

\author{
Jonathan Lacanlale \\ California State University, Northridge \\ jonathan.lacanlale.608@my.csun.edu
}

\author{
Premanand Kumar \\ University of California, San Diego \\ p8kumar@ucsd.edu
}

\author{
Kevin Yang \\ University of California, San Diego \\ khy009@eng.ucsd.edu
}

\author{
Arun Kumar \\ University of California, San Diego \\ arunkk@eng.ucsd.edu
}

\begin{abstract}
The paradigm of AutoML has created an opportunity to enable ML for the masses. Emerging industrial-scale cloud AutoML platforms aim to automate the end-to-end ML workflow. While many works have looked into automated feature engineering, model selection, or hyper-parameter search in AutoML, little work has studied a crucial step that serves as an entry point to this workflow: $M L$ feature type inference. The semantic gap between attribute types (e.g., strings, numbers) in databases/files and ML feature types (e.g., Numeric, Categorical) necessitates type inference. In this work, we formalize and standardize this task by creating the first ever benchmark labeled dataset, which we use to objectively evaluate existing AutoML tools. Our dataset has 9921 examples and a 9-class label vocabulary. Our labeled data also offers an alternative approach to automate this task than existing rule-based or syntax-based approaches: use ML itself to predict feature types. We collate a benchmark suite of 30 classification and regression tasks to assess the importance of type inference for downstream models. Empirical comparison on our labeled data shows that an ML-based approach delivers a lift of an average $14 \%$ and up to $38 \%$ in accuracy for identifying feature types compared to prominent industrial tools. Our downstream benchmark suite reveals that the ML-based approach outperforms existing industrial-strength tools for 47 out of 60 downstream models. We release our labeled dataset, models, and downstream benchmarks in a public repository with a leaderboard.
\end{abstract}

\section{ACM Reference Format:}

Vraj Shah, Jonathan Lacanlale, Premanand Kumar, Kevin Yang, and Arun Kumar. 2021. Towards Benchmarking Feature Type Inference for AutoML Platforms. In Proceedings of the 2021 International Conference on Management of Data (SIGMOD '21), June 20-25, 2021, Virtual Event, China. ACM, New York, NY, USA, 13 pages. https://doi.org/10.1145/3448016.3457274

\section{INTRODUCTION}

The paradigm of automated machine learning (AutoML) is beginning to help democratize machine learning for the masses [1]. Cloud

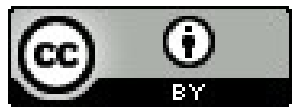

This work is licensed under a Creative Commons Attribution International 4.0 License. SIGMOD '21, fune 20-25, 2021, Virtual Event, China.

(C) 2021 Copyright held by the owner/author(s).

ACM ISBN 978-1-4503-8343-1/21/06.

https://doi.org/10.1145/3448016.3457274

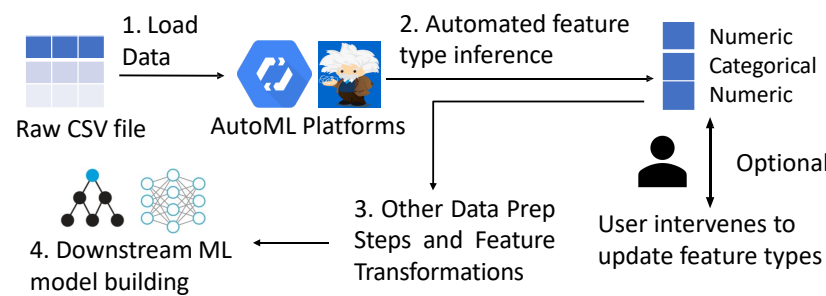

Figure 1: Typical workflow in AutoML platforms.

vendors have released AutoML platforms such as Google's Cloud AutoML [2] and Salesforce's Einstein [3] that build ML models on millions of datasets from thousands of small-and-medium enterprises automatically. The central goal of these platforms is to get an accurate model for the prediction task while achieving maximum possible automation of the end-to-end ML workflow, especially on structured data, including data transformations and feature engineering, as well as model building and hyperparameter tuning. The automation of these steps has been intensively studied in the $\mathrm{ML} /$ data mining $[1,4]$ and database communities [5, 6]. However, a crucial gateway step to this whole workflow has received much less attention so far: $M L$ feature type inference.

Datasets are typically loaded as files into the AutoML platforms. As Figure 1 illustrates, ML feature type inference is the very first step needed for ML over structured data. Features could be Numeric, Categorical, or something else, as shown in Figure 1. Determining the correct feature type is crucial for the whole workflow to work well: what data transformations to apply, how to extract features, and how to feed signals to the downstream models. For instance, if a column is inferred to be of type Timestamp, then several useful features such as day, month, and year are often extracted automatically for the downstream model. Thus, the accuracy of feature type inference is critical for the downstream model's accuracy, and in turn, the effectiveness of the entire ML platform.

Feature type inference is also performed automatically by many ML platforms, e.g. TransmogrifAI in Einstein [7], Tensorflow Data Validation (TFDV) in TensorFlow Extended [8], and AutoGluon from AWS [9]. But surprisingly, there is no objective evaluation to date of how good their automation of this task is. Thus, mistakes in their automated feature type inference can propagate and may degrade the workflow. For instance, consider what TFDV does on the illustrative dataset for a common ML task, customer churn 


\begin{tabular}{|c|c|c|c|c|c|c|c|}
\hline CustID & Gender & Salary & ZipCode & XYZ & Income & HireDate & Churn \\
\hline 1501 & 'F' & 1500 & 92092 & 005 & 'USD 15000' & '05/01/1992' & 'Yes' \\
\hline 1704 & 'M' & 3400 & 78712 & 003 & $' 25384 '$ & '12/09/2008' & 'No' \\
\hline
\end{tabular}

Figure 2: A simplified Customers data for churn prediction.

prediction in Figure 2. It wrongly calls many Categorical features with integer values as Numeric, e.g., ZipCode. This can cause the downstream model to produce garbage results. Moreover, Income is inferred as Categorical even though it has numbers embedded. Such issues can lead to loss of information and can potentially reduce the accuracy of the model, or even cause it to fail in some scenarios.

One might ask: Why cannot AutoML platform users manually verify their feature types? From our conversations with AutoML platform engineers at Salesforce and Google, we learned that their AutoML tools are used on over tens of thousands of datasets, adding up to millions of features in production settings. Forcing users to manually annotate features can lead to a tedious, slow, and errorprone process that also violates the promise of automation. Many domain users who may not have much ML expertise may not like the platform asking them to manually mark ML feature types. Thus, AutoML platform engineers prefer ever more accurate automation of this task. Clearly, this requires them to objectively measure the accuracy of their AutoML tool on the given task.

\subsection{This Paper's Focus}

Our Focus. We initiate work on benchmarking and objectively quantifying the task of $M L$ feature type inference in existing opensource industrial-strength AutoML tools. We formalize and standardize this task by creating a benchmark labeled dataset. This will enable an objective progress measurement, akin to ImageNet's role in vision [10]. Moreover, this will help objectively evaluate and improve AutoML platforms by enabling answers to key questions: How good are AutoML tools? How can one do better? How does the accuracy of type inference affect downstream ML model's accuracy?

Challenge. We first explain why feature type inference is hard to automate for existing rule-based or syntax-based systems. Datasets are typically loaded from RDBMSs, data lakes, or filesystems as flat CSV files into AutoML platforms. Thus, there exists a semantic gap between feature types for $M L$ and attribute types in databases/files. The latter tells us the syntactic datatypes of columns such as integer, real, or string. This semantic gap means reading syntax as semantics often leads to nonsensical results. For instance, consider Figure 2 again. Attributes such as CustID, Salary, and ZipCode are stored as integers, but only Salary is useful as Numeric. CustID is unique for every customer, hence it can not be generalized for ML. ZipCode is Categorical, even though it is stored as integers. In fact, this issue is ubiquitous in real-world datasets, since categories are often encoded as integers, e.g., item code, state code, etc.

Scope. Our focus is on relational/tabular data, which can be stored in any format (CSV, JSON, XML, etc.) and with any filesystems. Note that our focus is not to study any upstream processing steps that users might perform when they load their files into the AutoML tool. Also, our focus is not on feature engineering and transformation steps over the columns with the inferred types. We focus only on the ML feature type inference step. Admittedly, this is just one step in the entire end-to-end ML workflow, but we believe that studying this step in depth is critical to improve existing AutoML platforms, as we find that accurate type inference is critical for achieving high downstream model accuracy. Equally importantly, the predictions are more interpretable with accurate feature types.

\subsection{Benchmark Comparisons}

Our Labeled Dataset and Label Vocabulary. Creating labeled data for the task requires a common formalized label vocabulary, which is important to create because the dichotomy of Numeric vs. Categorical is not usually enough for categorizing feature types of raw columns. For instance, column HireDate in Figure 2 stores Date type values. Thus, we need more classes. We survey existing AutoML data prep tools and collect their feature type vocabulary into a common, practically useful set of labels that can be reused by any AutoML platform, as Figure 3 shows. We gather and handlabel the very first large meta-dataset for benchmarking feature type inference. Our dataset has 9921 columns from 1240 real data files from sources such as Kaggle and UCI ML repository. Our labeling process took about 90 man-hours across 5 months.

Current Limitation. We admit that files on Kaggle and UCI ML repository may not be representative of the truly "in-the-wild" dataset as it may have undergone some pre-processing. But, it is impractical for researchers to get access to large numbers of publicly releasable data from enterprises and organizations due to legal restrictions. Thus, Kaggle and UCI are the closest sources we have to the real-world data. We believe that our exploratory work is the first step in the direction of objectively evaluating AutoML tools. We hope that this work starts a conversation around enhancing such benchmark datasets.

Approaches to Type Inference. There are open-source tools such as Pandas [11], TransmogrifAI [7], TFDV [8], and AutoGluon [9] that automate this task. They all happen to be either rule-based or syntax-based. In contrast to prior approaches, our labeled dataset also presents an alternative approach to type inference: use ML itself to automate this task. We cast ML feature type inference as a multi-class classification problem and use ML models to bridge the semantic gap. We extract signals from raw data files that a typical data scientist may look at to identify the feature type. We summarize the signals in a feature set, which we use to build standard ML models on our labeled data. We empirically compare the ML-based approach enabled by our labeled data and existing public tools on our labeled test dataset.

Semantic Type Detection Tools. Recent tools such as Sherlock [12] and AutoType [13] perform column-level semantic type detection for automated data discovery and cleaning. The semantic type vocabulary of these tools is not directly usable for the AutoML setting because a semantic type can belong to multiple ML feature types. This is by design because the application motivations are different: semantic type detection tools are aimed at Business Intelligence (BI) tool users to browse attributes more easily, not AutoML users. Thus, it is complementary to our focus. To understand whether such tools can be ported to the AutoML setting, we use a rule-based 
approach to map Sherlock's semantic types to our vocabulary and evaluate it on our dataset.

Downstream Benchmark Suite. To understand the impact of the accuracy of ML feature type inference task on the downstream models, we create a downstream benchmark: 30 curated real-world datasets containing classification and regression tasks from diverse application domains such as healthcare, retail, sports, etc. The benchmark enables us to answer two key questions: (1) How does wrong type inference affect downstream performance? (2) How accurate are the downstream models delivered by the prior tools and the ML-based approach using our labeled data relative to performance with true feature types?

Empirical Evaluation and Analysis. An empirical comparison of different approaches on our labeled data shows that the MLbased approach delivers a lift of an average $14 \%$ and up to $38 \%$ in accuracy compared to existing tools for identifying feature types. We then evaluate and compare different ML models on our dataset. Overall, Random Forest outperforms the other models and achieves the best 9 -class accuracy of $92.6 \%$. We perform an ablation study on our ML models to characterize what types of features are useful.

Our empirical evaluation on the downstream benchmark suite shows that an ML-based approach using our labeled data delivers the most accurate downstream model against the prior tools for 47 out of 60 downstream models. In addition, we find that the wrong types inferred by existing tools often lead to a significant decrease in the downstream model's accuracy relative to their true accuracy. For instance, Pandas underperforms over truth in 45 out of 60 cases. Finally, we release a repository containing our labeled dataset, trained ML models, downstream benchmarks, and announce a leaderboard for community contributions.

In summary, our work makes four key contributions.

1. A new benchmark task and dataset. To the best of our knowledge, this is the first work to formalize and rigorously benchmark the task of ML feature type inference. We create the first large benchmark labeled datasets for this task with a readily practically useful 9-class label vocabulary.

2. Benchmarking alternate tools and approaches. Using our new data, we perform extensive empirical comparisons of open source and industrial (Auto)ML tools. Perhaps surprisingly, we find that even off-the-shelf ML models with standard featurization trained on our data significantly outperform all prior approaches.

3. Downstream benchmark suite. The curated benchmark offers evidence that the downstream model's performance can benefit by accurately determining feature types. We find that an ML model trained on our data for feature type inference often leads to more accurate downstream models than prior tools.

4. Real-world impact. Google collaborated with us to integrate our best performing ML models into TFDV to improve its inference of Categorical [14]. Google engineers are now reviewing it on internal benchmarks for adoption. AWS and OpenML [15] have also expressed interest in adopting our data and models for production use. Also, we release a public

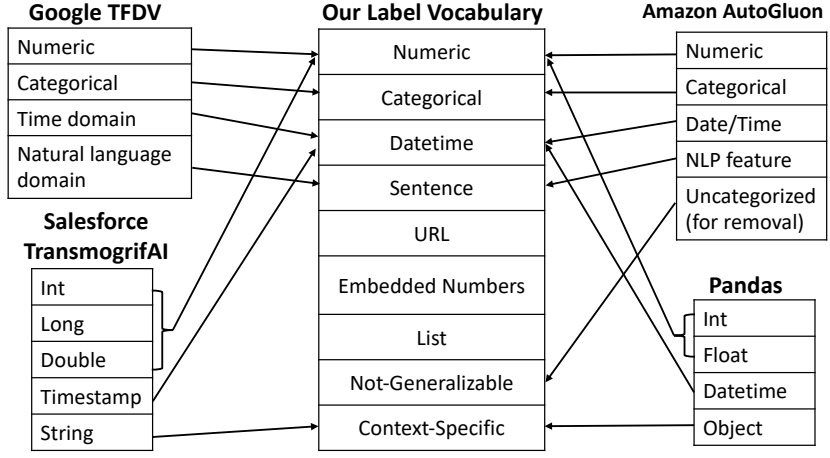

Figure 3: Feature type vocabulary mapping of TFDV, Pandas, TransmogrifAI, and AutoGluon to our vocabulary

competition on our labeled dataset to invite contributions to create/augment datasets, better featurizations, and models.

\section{OUR DATASET}

This section discusses our efforts in creating the labeled dataset. We discuss how we design the label vocabulary, the data sources, the signals we extract from the columns that enable us to inspect the columns succinctly, and the labelling process.

\subsection{Label Vocabulary}

Most ML models ultimately operate over only 2 (final) feature types: Numeric (continuous set) and Categorical (discrete set). Thus, each example (or column) has to be labelled as either of the two classes. However, we find that this bifurcation is not enough. This is because many other column types such as Date, URL, and Primary Keys are inevitable in the raw data file. Moreover, we find that the data file may not contain enough information to determine the feature type of a column, even for humans, e.g., column $X Y Z$ in Figure 2. Thus, we need more classes. We surveyed how the existing open source data prep tools such as Google's TFDV [8], TransmogrifAI in Salesforce Einstein [7], and AutoGluon from Amazon AWS [9] approach type inference and perform type-specific feature transformations. Figure 3 shows the feature type vocabulary of these tools. Inspired by this, we distill a common and practically useful set of labels for our vocabulary. We discuss the labels below.

(1) Numeric. These attributes are quantitative in nature and can directly be utilized as a Numeric feature for the downstream ML model. For instance, Salary is Numeric, while ID attributes such as CustID or integers representing encodings of discrete levels are not.

(2) Categorical. These attributes contain qualitative values that can directly be utilized as Categorical features for the downstream ML model. There are two major sub-classes: nominal and ordinal. Ordinal features have a notion of ordering among its values, while nominal do not. For instance, Year is ordinal, while ZipCode is nominal. Names and coded real-world entities from a known finite domain set are also Categorical. One often needs to alter the syntax of Categorical features for the downstream model, e.g., one-hot encoding in Scikit-learn or explicitly cast as a "factor" variable in R. 
(3) Datetime. This class represents attributes containing date or timestamp values, e.g., "7/11/2018”, and "21hrs:15min:3sec.” One may choose to extract custom features, either Numeric or Categorical or both through standard featurization routines. For instance, the month of the year can be Categorical, while time can be Numeric. Note that, such feature engineering decisions are not focus of this work since they are typically application-specific.

(4) Sentence. This class represents attributes containing textual values with semantic meaning. For instance, a passage of text may provide rich semantic information for a sentiment analysis application. One may choose to extract custom features, either Numeric or Categorical, or both through standard featurization routines. For instance, the AutoML platform developer can route such columns to an $n$-gram featurization routine or a routine to get Word2Vec embeddings from an English sentence for the downstream model. Again, we leave such downstream feature engineering decisions that come after type inference to the AutoML platform developer.

(5) URL. This class is for attributes whose values follow the URL standards [16]. This requires that the attribute values begin with a protocol followed by a sub-domain and a domain name. Any following information such as a file path is optional.

(6) Embedded Number. This class denotes attributes with "messy" syntax that preclude their direct use as Numeric or Categorical features. Thus, they require some form of processing before being used as as features. For instance, a number may be present along with string(s) denoting a measurement unit ("30 Mhz" or "USD 45 ") and/or special characters ("5,00,000"). In all cases, a number is typically extracted and the units are standardized (if applicable). One would typically use regular expressions or custom Python/R scripts for such extraction, e.g., converting “USD 45 ” to 45 .

(7) List. These attributes contain a list of items separated by a delimiter. One may write custom scripts to extract the domain of the list values and get new features for the downstream model.

(8) Not-Generalizable. An attribute in this class is a primary key in the table or has (almost) no informative values to be useful as a feature. Similarly, a column with only one unique value in the whole table offers no discriminative power and is thus useless. Such attributes are most unlikely to be used as features for the downstream model because they are not "generalizable." For example, CustID belongs to this class, since every future customer will have a new CustID. It is quite unlikely that one can get any useful features from it. Note that an attribute categorized as Not-Generalizable does not mean that it can never be useful for the downstream model. One may obtain some features from such attributes through more custom processing or domain knowledge. In contrast, even though attributes such as Income and Date may have all unique values in their columns, they are still generalizable. Thus, they belong to Embedded Numbers and Datetime respectively since it is highly likely that one can extract useful features from them.

(9) Context-Specific. This class is a catch-all for attributes that require human intervention either to determine their feature types and/or to inspect their values to build custom featurization routines.
The following examples illustrate this class. (1) Attributes wherein the data file does not have enough information even for a human to judge its feature type. Such columns typically have meaningless names, e.g., $X Y Z$ in Figure 2. Judging the feature type would require manually tracing down the provenance of how this column came to be using external "data dictionaries" maintained by the application or speaking to the data creator. (2) Attributes whose values require manual inspection for extracting useful features, e.g., JSON objects, geo-locations, addresses, or other complex objects that contain information dump about the data.

Our 9-class label vocabulary, while limited, is already practically useful for AutoML platforms. The label vocabulary can also give other insights to an AutoML platform developer. For instance, they could look for tables to join when faced with a large-domain Categorical feature such as ZipCode. They could route attributes marked as Embedded Numbers or Datetime to suitable Python/R scripts. Moreover, they could dispatch the columns that are marked Not-Generalizable for any missing values or errors in data entry to appropriate libraries. Finally, they could prompt for user intervention on only the columns that are marked Context-Specific. This can reduce user time spent on annotation significantly.

\subsection{Data Sources}

We gather 1240 CSV data files from sources such as Kaggle and UCI ML repository. Each column of the CSV file is just one example for our task. We obtain 9921 examples from all data files. Note that we do not always use all the columns from a single data file for labeling. We explain this in Section 2.4. Kaggle and UCI ML are the largest public data sources that are closest to real-world datasets. However, we note a caveat that the files on Kaggle and UCI ML may have undergone some pre-processing. It is almost impossible for researchers to get access to large numbers of truly "in-the-wild" data from enterprises and other organizations and make them publicly available due to legal restrictions. But the crux of our point in this paper is this: even on data files from Kaggle and UCI, existing open-source and industrial tools yield relatively poor accuracy compared to the ML models trained on our data (Section 4.2). Thus, we believe our work is a promising start towards objectively evaluating AutoML platforms.

\subsection{Base Featurization}

To identify the feature type of a raw column, a human data scientist may look at the column name, some sample values in the column, and even descriptive stats about the column. For instance, just by reading the attribute name, ZipCode, an interpretable string, a human can tell its feature type is Categorical. Thus, we represent the columns in a more concise way such that it emulates what a typical data scientist may look at to determine the feature type. We call this step Base Featurization. We extract the following base features for every column in the raw data file.

(1) Column name. We extract the column name as it can give crucial semantic clues for the feature type.

(2) Column values. A human would typically inspect some values in the column to make sure they make sense. For instance, values with decimal points are likely to mean Numeric features, while 
values with delimiters are likely lists. Thus, we extract 5 randomly sampled distinct attribute values from the column. We choose 5 because we think it is a reasonable number for a human to understand the column and determine the feature type when doing manual labeling (Section 2.4). However, this number can very well be higher or lower. It can be even tuned when building an ML model or a heuristic. In fact, from the ablation study of the ML models built on the base features, we find that even one or two sample values may be good enough to build an accurate model (Table 2).

(3) Descriptive statistics. Finally, a human would look at some descriptive stats about the column. For instance, if the human finds that all values in the column are NaNs, then they might classify the column as Not-Generalizable. Considering this, we extract 25 descriptive stats for a column such as the total number of values, the absolute number and \% of NaNs relative to total values, the absolute number and \% of distinct values relative to total values, mean, and standard deviation. We provide the complete list of these features in the technical report [17].

Each column in the raw data file is an example in the new base featurized file and we manually label every example of the base featurized file. The base featurization step also helps to deliver an ML-based approach to type inference (Section 4.3).

\subsection{Labelling Process}

We first use base featurized columns from 360 source files to label them in one of the nine classes. But, we find that they only contain a small handful of examples for the classes: URL, List, Sentence, Embedded Number, and Datetime. Thus, we use an additional 880 source data files to only label the examples for the under-represented classes. We extract these examples from additional sources as we did not want to create a heavily skewed class label distribution to get good confidence on all classes. Note that augmenting classes where the number of examples is under-represented is a common practice in the ML literature [18-21]. Since our benchmark contains multiple class-level accuracy metrics (discussed in Section 4.1), inspecting them can provide more confidence with the class predictions. Furthermore, we find that many data files have a series of column names such as $x y z 1, x y z 2$, and so on. Thus, we drop the columns with a repeating series of names.

To reduce the cognitive load of labelling, we follow the following process. Initially, we manually label 500 examples. We then use Random Forest with 100 estimators to perform 5-fold nested crossvalidation $(\mathrm{CV})$. The model achieves a classification accuracy of around $74 \%$ on the test set (average across 5 folds). We use this model to predict a class label on all of the 9921 examples. We then group all the examples by these predicted labels and inspect all of them manually. Such grouping helps reduce the cognitive load caused by class context switches during labeling. The labeling process took about 90 man-hours across 5 months.

We also tried to crowdsource labels on the FigureEight platform but abandoned this effort because the label quality was too low across two trial runs. We suspect such high noise arises because this task is too technically nuanced for lay crowd workers relative to popular crowdsourcing tasks like image recognition. Devising better crowdsourcing schemes for our task with lower label noise is an avenue for future work. We summarize our crowdsourcing results in the technical report [17].

\subsection{Data Statistics}

The distribution of class labels in our labeled dataset is: Numeric (36.6\%), Categorical (23.3\%), Datetime (7\%), Sentence (3.9\%), URL (1.5\%), Embedded Number (5.7\%), List (2.4\%), Not-Generalizable (10.6\%), and Context-Specific (8.9\%). We provide a complete breakdown of the cumulative distribution by class for different descriptive statistics in the technical report [17].

\section{APPROACHES COMPARED}

In this section, we discuss the different approaches to type inference. We first discuss existing open-source tools that all happen to be either rule-based or syntax-based. We then briefly discuss an intuitive rule-based baseline to check if a set of rules can accurately capture our labeled dataset. Finally, we explain how our labeled dataset is used to build ML models.

\subsection{Existing Tools}

Figure 3 shows the feature type vocabulary of these tools and how they map to our label vocabulary.

Tensorflow Data Validation (TFDV). TFDV is a tool to analyze and transform ML data in TensorFlow Extended (TFX) pipeline [8]. TFDV uses heuristics to infer ML feature types such as numeric, categorical, time or date domain, or natural language text from the descriptive statistics about the column. The users can then review the inferred feature types and can update them manually.

Pandas. Pandas is a Python library that provides tools for data analysis and data transformations. It infers syntactic types such as integer, float, or object [11]. It also provides a utility function that can check the column for the datetime type.

TransmogrifAI. This is an AutoML library for structured data in Salesforce's AutoML platform called Einstein [7]. TransmogrifAI supports rudimentary automatic feature type inference over primitive types such as Integer, Long, Double, Timestamp, and String. It also has an extensive vocabulary for feature types such as email, phone numbers, zipcodes, etc. However, users have to manually specify these types for their data.

AutoGluon-Tabular. AutoGluon is an end-to-end AutoML framework from AWS [9]. It classifies each column into numeric, categorical, date/time, text, or columns that needs to be discarded because they can't be classified into any of the classes.

Sherlock. Sherlock [12] is a distantly-supervised deep-learningbased tool that identifies 78 semantic types such as Age, Code, Duration, etc. But the semantic types are not directly usable for AutoML because the same semantic type can span different ML feature types. For instance, Duration type can be either Numeric (e.g., time elapsed in seconds), Categorical (e.g., time duration belonging to a discrete set), Datetime (e.g. the exact timestamp), or even Sentence (e.g., duration mentioned in words).

We find that out of 78 semantic types, 55 types can be uniquely mapped to one single class of our label vocabulary. The number of types that are mapped to 2, 3, and 4 classes of our label vocabulary are 18,3 , and 2 , respectively. We release the mapping between 


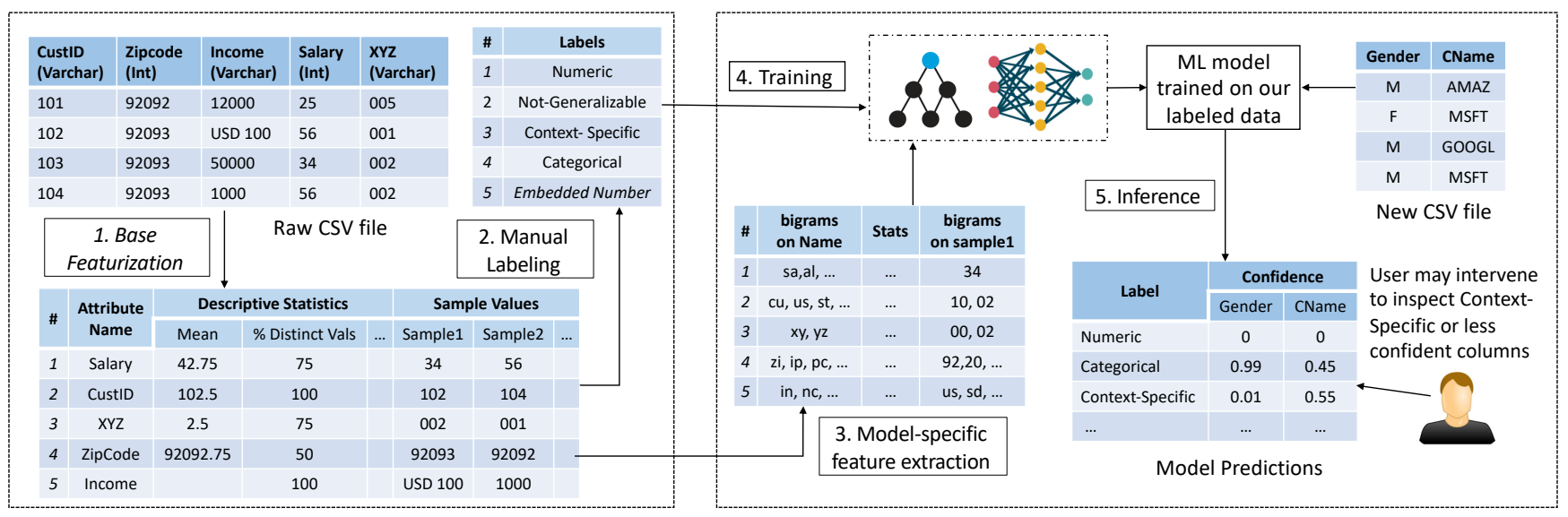

Figure 4: Workflow showing our labeling process and how our data is used for ML-based feature type inference.

Sherlock semantic types and our label vocabulary in the technical report [17]. We use a rule-based approach on top of Sherlock to identify one single feature type given a column. We discuss examples to illustrate how we map semantic types to our feature types in the technical report [17].

\subsection{Rule-based Baseline}

We use this approach to validate if a set of rules can accurately represent our labeled dataset. We write 11 rules to capture all the classes using a flowchart-like structure. We provide two examples below. (1) To identify List, non-empty sample values are matched with a regular expression based check of a series of characters separated by a type of delimiter such as ; I , etc. (2) If either of the \% of NaNs or \% of unique values in the column are greater than $99.99 \%$ then we mark it as Not-Generalizable. We describe the complete rule-based approach in the technical report [17].

\subsection{ML-based Approach using our Data}

As shown in Figure 4, we use our labeled data to build standard ML models. Base Featurization is a common step for all ML models. Some ML models cannot operate on the raw characters of attribute names or sample values. Thus, we extract hand-crafted feature sets from the attribute names and sample values. We then train several classical ML models, $k$-NN with a distance function tuned for our task, and a CNN. Finally, the pre-trained model is used to infer feature types for columns in an "unseen" CSV file. At the scale of AutoML platforms where there are potentially millions of columns, human intervention can be costly and slow. The models output predictions and the corresponding confidence scores for each class. Thus, an ML-based approach allows users to intervene to prioritize their effort towards Context-Specific types or columns with low confidence scores that may need more human attention.

3.3.1 Feature Extraction. The attributes with similar names can likely belong to the same class. For instance, both attributes temperature_jan and temperature feb are Numeric. Similarly, knowing that the sequence of characters are numbers followed by a "/," can give an indication of Datetime. Based on these intuitions, we extract an $n$-gram feature set from the attribute names and sample values.
Notation. We denote the descriptive stats by $\mathbf{X}_{\text {stats }}$, the attribute name by $\mathbf{X}_{\text {name }}$, and randomly sampled attribute values by $\mathbf{X}_{\text {sample }}$ (first sampled value referred to as $\mathbf{X}_{\text {sample } 1}$ and similarly for other values). We leverage the commonly used bigram features on the attribute name (denoted by $\left.\mathbf{X} 2_{\text {name }}\right)$ and sample value ( $\left.\mathbf{X} 2_{\text {sample }}\right)$.

3.3.2 Classical ML models. We consider classical models: Logistic Regression, RBF-SVM, and Random Forest. Note that they cannot operate on raw characters of attribute names or sample values.

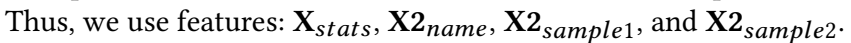
For scale-sensitive models such as RBF-SVM and logistic regression, we standardize $\mathbf{X}_{\text {stats }}$ to have mean 0 and standard deviation 1 .

3.3.3 Nearest Neighbor. Most implementations of $k$-NN use a simple Euclidean distance. But, we can adapt the distance function for the task at hand by defining the weighted distance function as:

$$
d=E D\left(X_{\text {name }}\right)+\gamma \cdot E C\left(X_{\text {stats }}\right)
$$

Here, $E D$ (resp. $E C$ ) is the edit distance (resp. euclidean distance) between $X_{\text {name }}$ (resp. $X_{\text {stats }}$ ) of a test example and a training example. $\gamma$ is the parameter that needs to be tuned during training.

3.3.4 CNN. Inspired by the success of CNN on short text classification tasks [22,23], we leverage a character-level CNN for our task. The network takes attribute name, descriptive stats, and sample values as input and outputs the class from the label vocabulary. We present the architecture and layers of $\mathrm{CNN}$ in the tech report [17].

\section{EMPIRICAL STUDY AND ANALYSIS}

We now empirically compare the industrial open source tools and ML models on the accuracy of type inference. This is the very first empirical comparison of this sort of these tools, thanks to our new benchmark labeled dataset. The headline result is that our ML models substantially surpass these prior tools on test accuracy.

\subsection{Methodology and Setup}

Methodolody. We partition our labeled dataset into a train and held-out test set with 80:20 ratio. We perform 5-fold nested crossvalidation of the train set, with a random fourth of the examples in a training fold being used for validation during hyper-parameter 
Table 1: Binarized class-specific accuracy of different approaches on our benchmark labeled held-out test dataset. The bold fonts highlight the most accurate approach/model per class.

\begin{tabular}{|c|c|c|c|c|c|c|c|c|c|c|}
\hline \multirow{2}{*}{ Feature Type } & \multirow{2}{*}{ Metric } & \multicolumn{4}{|c|}{ Open-source Industrial Tools } & \multirow{2}{*}{$\begin{array}{c}\text { Sherlock + } \\
\text { Rules }\end{array}$} & \multirow{2}{*}{$\begin{array}{c}\text { Baseline } \\
\text { Rule-based }\end{array}$} & \multicolumn{3}{|c|}{ Models trained on our data } \\
\hline & & TFDV & Pandas & TransmogrifAl & AutoGluon & & & Log Reg & $\mathrm{CNN}$ & Rand Forest \\
\hline \multirow{3}{*}{ Numeric } & Precision & 0.657 & 0.614 & 0.605 & 0.646 & 0.599 & 0.773 & 0.909 & 0.929 & 0.934 \\
\hline & Recall & 1 & 1 & 1 & 1 & 0.359 & 0.946 & 0.943 & 0.941 & 0.984 \\
\hline & Accuracy & 0.814 & 0.776 & 0.767 & 0.805 & 0.683 & 0.882 & 0.946 & 0.953 & 0.97 \\
\hline \multirow{3}{*}{ Categorical } & Precision & 0.396 & \multirow{3}{*}{ - } & \multirow{3}{*}{-} & 0.667 & 0.311 & 0.577 & 0.808 & 0.846 & 0.913 \\
\hline & Recall & 0.652 & & & 0.534 & 0.707 & 0.457 & 0.884 & 0.928 & 0.943 \\
\hline & Accuracy & 0.691 & & & 0.831 & 0.567 & 0.798 & 0.925 & 0.945 & 0.966 \\
\hline \multirow{3}{*}{ Datetime } & Precision & 0.985 & 0.956 & 1 & 1 & 0.89 & 0.559 & 0.951 & 0.925 & 0.945 \\
\hline & Recall & 0.475 & 0.915 & 0.454 & 0.844 & 0.801 & 0.135 & 0.972 & 0.965 & 0.972 \\
\hline & Accuracy & 0.962 & 0.991 & 0.961 & 0.989 & 0.979 & 0.931 & 0.994 & 0.992 & 0.994 \\
\hline \multirow{3}{*}{ Sentence } & Precision & 0.472 & \multirow{3}{*}{ - } & \multirow{3}{*}{-} & 0.516 & 0.354 & 1 & 0.913 & 0.725 & 0.865 \\
\hline & Recall & 0.457 & & & 0.902 & 0.554 & 0.043 & 0.793 & 0.804 & 0.902 \\
\hline & Accuracy & 0.951 & & & 0.956 & 0.932 & 0.956 & 0.987 & 0.977 & 0.989 \\
\hline \multirow{3}{*}{$\begin{array}{l}\text { Not- } \\
\text { Generalizable }\end{array}$} & Precision & \multirow{3}{*}{-} & \multirow{3}{*}{ - } & \multirow{3}{*}{-} & 0.465 & 0.692 & 0.216 & 0.732 & 0.81 & 0.934 \\
\hline & Recall & & & & 0.53 & 0.042 & 0.507 & 0.732 & 0.66 & 0.86 \\
\hline & Accuracy & & & & 0.883 & 0.893 & 0.747 & 0.947 & 0.937 & 0.978 \\
\hline \multirow{3}{*}{$\begin{array}{l}\text { Context- } \\
\text { Specific }\end{array}$} & Precision & \multirow{3}{*}{ - } & 0.08 & 0.074 & \multirow{3}{*}{-} & 0.192 & 0.211 & 0.747 & 0.741 & 0.859 \\
\hline & Recall & & 0.295 & 0.295 & & 0.168 & 0.195 & 0.621 & 0.663 & 0.705 \\
\hline & Accuracy & & 0.609 & 0.582 & & 0.851 & 0.853 & 0.944 & 0.946 & 0.961 \\
\hline
\end{tabular}

tuning. We use a standard grid search for hyper-parameter tuning. We describe the grids in the technical report [17]. We also did a 5-fold leave-data file out cross-validation to "stress-test" the ML models for new data files. The raw data files were split into 60:20:20 train, validation, and test partitions where each partition has all columns of a particular data file. Thus, the test partition has columns of the raw data files that the model has not seen before. The trends of the leave-data file out approach are similar to the former approach; so, we discuss its results in the technical report [17].

Experimental Setup. We use CloudLab [24] with custom OpenStack profile running Ubuntu 18.04 with 10 Intel Xeon cores and 192GB of RAM. For TFDV, Transmogrifai, AutoGluon, and Pandas, we use version number $0.22 .2,0.7 .0,0.0 .11$, and 0.25 .3 respectively.

Metrics. Our key metric is prediction accuracy for the 9-class task. We also use class-specific binarization metrics such as precision, recall, F1 score, and confusion matrix.

\subsection{Comparison of All Approaches}

We compare ML models trained on our dataset against open-source tools on our held-out test data. Figure 3 showed the feature type vocabulary of these tools and how they map to our vocabulary. Since none of these tools support our full 9-class vocabulary, we report results on binarization of our classes: Numeric vs. all Non-Numeric, Categorical vs. all Non-Categorical, and similarly for others.

Results. Table 1 presents the precision, recall, and overall $2 \times 2$ diagonal accuracy results of all approaches on our benchmark labeled held-out test set ${ }^{1}$. We report F1 score and full confusion matrices in the technical report [17]. We present the results in-depth below.

(1) We see that the ML models achieve significantly higher accuracy than all industrial tools across the board for all feature types. For instance, a lift of $28 \%$ and $14 \%$ in accuracy in predicting Categorical compared to TFDV and AutoGluon respectively. Of all approaches, Random Forest achieves the highest accuracy in inferring the types.

(2) Interestingly, all the existing tools have a high recall on Numeric but very low precision. This is because their heuristics are syntactic, which leads them to wrongly classify many Categorical features such as ZipCode as Numeric. The ML models have a slightly lower recall on Numeric. This is because, with many features thrown, they get slightly confused and could wrongly predict a Numeric type as

\footnotetext{
${ }^{1}$ Since publication, we have released version 2 of our labeled dataset where 32 examples are relabeled after feedback on Github [25]. We find only minor changes in the results without altering any of our trends, conclusions, or takeaways discussed here. Note that our labeled dataset is a living public repository on Github which we anticipate to grow in the future. Please refer to our public repository for the up-to-date results.
} 
Table 2: Full 9-class test accuracy of the ML models trained on our data with different feature sets. $X_{\text {name }}^{*}, X_{\text {sample }}^{*}, X_{\text {sample } 2}^{*}$ denote bigram features $\left(X 2_{\text {name }}, X 2_{\text {sample } 1}, X 2_{\text {sample } 2}\right)$ for classical $M L$ models and raw character-level features $\left(X_{\text {name }}, X_{\text {sample } 1}, X_{\text {sample } 2}\right)$ for $\mathrm{CNN}$ and $k$-NN. The bold fonts highlight the most accurate feature set for that model.

\begin{tabular}{|c|c|c|c|c|c|c|c|c|c|}
\hline & $X_{\text {stats }}$ & $\mathrm{X}^{*}$ name & $\mathrm{X}_{\text {sample1 }}^{*}$ & $\mathrm{X}_{\text {stats, }}, \mathrm{X}^{*}{ }_{\text {name }}$ & $\mathrm{X}_{\text {stats, }} \mathrm{X}_{\text {sample } 1}$ & $\mathrm{X}^{*}{ }_{\text {name, }} \mathrm{X}^{*}{ }_{\text {sample } 1}$ & $\mathrm{X}^{*}{ }_{\text {sample1 } 1} \mathrm{X}_{\text {sample } 2}^{*}$ & $\begin{array}{c}\mathrm{X}_{\text {stats, }} \mathrm{X}^{*}{ }_{\text {name, }} \\
\mathrm{X}^{*}{ }_{\text {sample } 1}\end{array}$ & $\begin{array}{c}\mathrm{X}_{\text {stats, }} \mathrm{X}^{*}{ }_{\text {name, }} \\
\mathrm{X}^{*}{ }_{\text {sample1 }} \mathrm{X}^{*}{ }_{\text {sample2 }}\end{array}$ \\
\hline $\begin{array}{l}\text { Logistic } \\
\text { Regression }\end{array}$ & 0.6862 & 0.7293 & 0.6603 & 0.8428 & 0.7763 & 0.8043 & 0.7144 & 0.8578 & 0.8643 \\
\hline RBF-SVM & 0.8213 & 0.777 & 0.6521 & 0.8724 & 0.7845 & 0.8159 & 0.7131 & 0.8761 & 0.8712 \\
\hline $\begin{array}{l}\text { Random } \\
\text { Forest }\end{array}$ & 0.9121 & 0.7785 & 0.6657 & 0.9259 & 0.8956 & 0.8346 & 0.7374 & 0.9216 & 0.9096 \\
\hline CNN & 0.6809 & 0.8019 & 0.6805 & 0.8692 & 0.7965 & 0.8655 & 0.7763 & 0.8788 & 0.8701 \\
\hline k-NN & 0.8605 & 0.7839 & - & 0.8796 & - & - & - & - & - \\
\hline
\end{tabular}

non-numeric. But, the ML models have much higher precision and high overall accuracy.

(3) Heuristics for identifying Datetime by all the existing tools have high precision, even higher than the ML models. However, their rules do not capture many Datetime type instances (e.g., an attribute named BirthDate "19980112"); thus, they have a much lower recall.

(4) The heuristic rules of AutoGluon and TFDV are largely dependent upon the number of words in a string for accurately inferring Sentence type. Thus, a column with most of its values having a large number of words will likely get inferred as Sentence by these tools. However, a Categorical or Context-Specific column (e.g., containing JSON object) can satisfy the criteria provided by the rules. Thus, AutoGluon and TFDV have low precision on Sentence. On the other hand, the ML-based approaches have much higher precision.

Other Commercial Tools. There exist other commercial tools that also automate the ML feature type inference task such as Google AutoML Tables [26], DataRobot [27], and Trifacta [28]. However, since these systems are closed source, we do not know how these tools work. It is also hard to evaluate their accuracy because: (1) DataRobot has no public/free trial version of their platform. We got no response to our demo request. (2) AutoML Tables and Trifacta only offer GUI-based usage where users must upload the raw CSV files manually to identify the feature types. Both these tools do not provide any programmatic way for evaluation. So, we cannot evaluate their accuracy automatically. We manually uploaded 5 CSV files from our raw data. All 15 categoricals encoded as integers were (wrongly) classified as Numeric by both tools. Since it is hard to draw any generalizable conclusion if these tools have the same issues as TFDV, AutoGluon, and TransmogrifAI, we leave it to future work to assert this more systematically.

\subsection{Comparison of ML-based Approaches}

Rule-based Baseline. The 9-class classification accuracy on the held-out test set is only $54 \%$. We observe that this approach achieves $95 \%$ and $46 \%$ recall in classifying Numeric and Categorical respectively. The recall for Categorical is low because a category encoded as a number is wrongly classified as Numeric. Admittedly, our rules are not exhaustive and one can always come up with more rules to improve the accuracy. However, writing rules for every little corner case is excruciating and will likely never be comprehensive.

Sherlock. Sherlock with a rule-based approach that maps their semantic types to our label vocabulary has an accuracy of just $42 \%$. This is because their semantic type vocabulary is not suitable towards identifying ML feature types. The number of Sherlock semantic types (out of 78) that are mapped to ML feature types are: 14 to Numeric, 50 to Categorical, 4 to Datetime, 7 to Sentence, 11 to Embedded Number, 2 to List and Not-Generalizable, and 18 to Context-Specific. Since Categorical type occur most frequently, more examples in our labeled dataset are disproportionately confused with this feature type. For instance, many integer Numeric attributes are confused with semantic types that often contains discrete set of integers (such as Credit and Class). Interestingly, Sherlock has a high precision of $89 \%$ in identifying Datetime correctly, even with just 4 semantic type mapped to Datetime.

Classical ML Models. Table 2 presents the 9-class accuracy results of the classical ML models using different feature sets ${ }^{1}$. We present the 5-fold held-out train and validation accuracy in the technical report [17]. For logistic regression, we see that the descriptive stats alone are not enough, as it achieves an accuracy of just $69 \%$ on the held-out test set. But, for RBF-SVM and Random Forest, the accuracy with stats alone is already $82 \%$ and $91 \%$ respectively. Incorporating bigrams of the attribute name into logistic regression leads to a whopping $15 \%$ lift in accuracy. However, adding more sample values does not give any rise in accuracy, except for logistic regression. Overall, Random Forest achieves the best 9-class accuracy of $93 \%$ using bigrams on the attribute name along with descriptive statistics.

CNN and Nearest Neighbor. Table 2 also shows the CNN and $k$-NN accuracy ${ }^{1}$. We see that with just $X_{\text {name }}$, the $\mathrm{CNN}$ accuracy is already $82 \%$. The descriptive stats lift the accuracy further by $8 \%$. We find that sample values are not that useful, yielding only a minor lift. With $k$-NN, we observe that with only Euclidean distance on descriptive statistics, the accuracy is already at $86 \%$. The edit distance on the attribute name approach achieves an accuracy of $78 \%$. Finally, with our weighted edit distance function from Section $4.4, k$-NN achieves a high $88 \%$ accuracy. 
Table 3: Examples of errors made by RandomForest. $\mathrm{Nu}$ meric (NU), Categorical (CA), Datetime (DT), Sentence (ST), Not-Generalizable (NG), Embedded Number (EN), URL, List (LST), and Context-Specific (CS) are feature types.

\begin{tabular}{|c|c|c|c|c|c|c|c|}
\hline$\#$ & $\begin{array}{c}\text { Attribute } \\
\text { Name }\end{array}$ & $\begin{array}{c}\text { Sample } \\
\text { Value }\end{array}$ & $\begin{array}{c}\text { Total } \\
\text { Values }\end{array}$ & $\begin{array}{c}\% \text { Distinct } \\
\text { Values }\end{array}$ & $\%$ NaNs & Label & $\begin{array}{c}\text { RF } \\
\text { Prediction }\end{array}$ \\
\hline A & s1p1c2area & 50 & 9597 & 3.6 & 45.2 & NU & CS \\
\hline B & Tenure Status & $\begin{array}{c}\text { Own house, } \\
\text { rent lot }\end{array}$ & 41544 & 0.02 & 0 & CA & ST \\
\hline C & End & March 4, 1797 & 45 & 97.8 & 2.2 & DT & EN \\
\hline D & Name & $\begin{array}{c}\text { Battle of } \\
\text { Riverrun }\end{array}$ & 38 & 100 & 0 & ST & NG \\
\hline E & \%White & $18.90 \%$ & 192 & 58.9 & 0 & EN & CA \\
\hline F & Countries & ru; uk; mx & 1359 & 32.9 & 46.3 & LST & EN \\
\hline G & q19TalToolResumeScreen & \#NULL! & 25090 & 0.008 & 6 & NG & CA \\
\hline H & Livshrmd & 151 & 9597 & 1.17 & 42.3 & CS & NU \\
\hline
\end{tabular}

\subsection{Analysis of Errors}

We now explain the behavior of the best performing Random Forest on our held-out test dataset (shortened henceforth as "OurRF") by inspecting the raw datatype of the column values. Table 3 shows examples of columns and the corresponding prediction made by OurRF. We present the full confusion matrix of the predicted class by OurRF vs actual data type of the attribute value in the technical report [17]. We intuitively explain the errors by class below.

Numeric and Context-Specific. We see that OurRF is less likely to misclassify a Numeric attribute whose values are floats or negative numbers compared to integers. We observe that with integers, OurRF gets most confused with Context-Specific class, e.g., s1p1c2area (Table 3 example(A)). This is possibly because of the non-sensical attribute name. Similarly, Context-Specific integers are most commonly misclassified with Numeric (Table 3 example(H)).

Categorical and Not-Generalizable. When the sample values are strings with more than one token, OurRF is more likely to misclassify Categorical as Sentence or Context-Specific (Table 3 example(B)). Not-Generalizable types are often confused with Categorical. For instance, q19TalTool-ResumeScreen (Table 3 example $(\mathrm{G})$ ) has only 2 values in its domain: "NULL!" and "ResumeScreen." However, OurRF treats "NULL!" as a separate category. Thus, OurRF is lacking in its semantic understanding ability of sample values.

Other types. We find that our model achieves high precision and recall in inferring other types such as Datetime and URL. In addition, List types are often confused with Embedded Number (Table 3 example(C)) even though there is no number available for extraction. This can be due to few available training examples for List type.

\subsection{Prediction Runtimes and Extensions}

We evaluate the running time of ML models in the online phase, i.e, to make predictions on a new column. This involves base featurization, model-specific feature extraction (only needed for the classical models), and inference time. The measurements were made on the test set and averaged. All the models finish in under $0.2 \mathrm{sec}$ per column. For classical models, the additional feature extraction dominates the overall runtime. Since SVM and $k$-NN are distance-based
Table 4: (A) Type Inference accuracy on 30 downstream datasets. (B) Number of downstream datasets where tools underperform, match, or outperform the ground truth downstream performance or the best performing tool. OurRF is the Random Forest for type inference trained on our data. LR denotes downstream linear model (Logistic/Linear regression) and RF denotes downstream Random Forest.

\begin{tabular}{|l|c|c|c|c|}
\hline (A) & Pandas & TFDV & AutoGluon & OurRF \\
\hline Column Coverage & 300 & 535 & 553 & 566 \\
\hline $\begin{array}{l}\text { Type inference accuracy } \\
\text { given coverage }\end{array}$ & $90.3 \%$ & $75 \%$ & $71.4 \%$ & $91.2 \%$ \\
\hline
\end{tabular}

\begin{tabular}{|l|c|c|c|c||c|c|c|c|}
\hline \multirow{2}{*}{ (B) } & \multicolumn{4}{|c||}{ Logistic Regression } & \multicolumn{4}{c|}{ Random Forest } \\
\cline { 2 - 10 } & PD & TFDV & AGL & OurRF & PD & TFDV & AGL & OurRF \\
\hline Underperform truth & 23 & 18 & 19 & 11 & 21 & 17 & 16 & 9 \\
\hline Match truth & 6 & 10 & 10 & 16 & 7 & 11 & 12 & 19 \\
\hline Outperform truth & 1 & 2 & 1 & 3 & 2 & 2 & 2 & 2 \\
\hline $\begin{array}{l}\text { Best performing } \\
\text { tool for a dataset }\end{array}$ & 9 & 11 & 10 & 23 & 10 & 14 & 16 & 24 \\
\hline
\end{tabular}

methods, they have the highest runtime. Overall, $\mathrm{CNN}$ is the fastest. We present the time breakdown in the technical report [17].

Our benchmark and ML-based approach can be easily extended to support new additional types, including semantic types [12]. We showcase the the effort needed for this extension in the context of two semantic data types that are commonly used in BI applications: Country and State. We find that the overhead of supporting these additional types in terms of programming cost, feature engineering cost, and labeling cost is minimal to almost none. We present the complete discussion in the technical report [17].

\section{DOWNSTREAM BENCHMARK SUITE}

To complete the loop on type inference, we now empirically study if doing feature type inference accurately is essential for downstream model accuracy. Thus, we verify if there are cases where doing wrong type inference may improve, reduce, or match the downstream accuracy relative to true feature types. From Section 4.3, we saw that type inference accuracy is highest for the Random Forest (OurRF) among all ML-based approaches. Thus, we compare the OurRF against the industrial and open source tools on a suite of downstream tasks we collected and curated.

\subsection{Datasets}

The impact of type inference is dependent on the dataset and the downstream prediction task. Since there are unboundedly many datasets and downstream tasks, for the sake of tractability we got 30 "unseen" datasets from Kaggle, UCI ML repository, and OpenML [15] for evaluation. Since classification tasks are more common in practice, we got 25 datasets for such tasks, and 5 for regression tasks. Table 5 presents the downstream datasets with descriptions such as their number of columns, target classes, and different feature types and attribute types they contain. We ensure representation of various combinations of feature types with many different data types (ints, floats, string, dates, timestamps, and even primary keys). We did not cherry-pick a dataset to particularly suit one approach 
Table 5: Accuracy comparison of downstream models using inferred types from Random Forest trained on our labeled data (OurRF) against Pandas (PD), TFDV, and AutoGluon (AGL), relative to accuracy with true feature types. Datasets involve (A) Classification tasks with accuracy metric (B) Regression tasks with RMSE metric. Numeric(NU), Categorical(CA), Datetime (DT), Sentence(ST),Not-Generalizable (NG), Embedded Number (EN), URL, List (LST), and Context-Specific (CS) are feature types. |A| is the number of columns/attributes in that dataset. $|\mathrm{Y}|$ is the number of target classes. PK denote primary keys. ${ }^{*}$ denotes the cases where OurRF prediction is either $E N$ or $C S$, where user intervention can help improve model accuracy or generalization.

\begin{tabular}{|c|c|c|c|c|c|c|c|c|c|c|c|c|c|c|c|c|c|}
\hline \multirow{2}{*}{\multicolumn{2}{|c|}{ (A) $\begin{array}{c}\text { Feature } \\
\text { Types }\end{array}$}} & \multirow{2}{*}{\multicolumn{2}{|c|}{$\begin{array}{c}\text { Raw Attribute } \\
\text { Types }\end{array}$}} & \multirow{2}{*}{\multicolumn{2}{|c|}{ Dataset }} & \multirow{2}{*}{$|A|$} & \multirow{2}{*}{$|Y|$} & \multicolumn{5}{|c|}{ Logistic Regression } & \multicolumn{5}{|c|}{ Random Forest } \\
\hline & & & & & & & & Truth & PD & TFDV & AGL & OurRF & Truth & $P D$ & TFDV & AGL & OurRF \\
\hline \multirow{2}{*}{\multicolumn{2}{|c|}{ NU }} & \multicolumn{2}{|l|}{ Int, Float } & \multicolumn{2}{|c|}{ Cancer } & 9 & 2 & 60.8 & +0 & +0 & +0 & +0 & 66.7 & +0 & +0 & +0 & +0 \\
\hline & & \multicolumn{2}{|l|}{ Int } & \multicolumn{2}{|c|}{ Mfeat } & 216 & 10 & 92.5 & +0 & +0 & +0 & -2.7 & 91.8 & +0 & +0 & +0 & -2.3 \\
\hline \multirow{7}{*}{\multicolumn{2}{|c|}{ CA }} & \multicolumn{2}{|l|}{ String } & \multicolumn{2}{|c|}{ Nursery } & 8 & 5 & 92.8 & -0.9 & +0 & +0 & +0 & 98.2 & -3.9 & +0 & +0 & +0 \\
\hline & & \multicolumn{2}{|l|}{ String } & \multicolumn{2}{|c|}{ Audiology } & 69 & 24 & 73 & -1.3 & +0 & -1.3 & +0 & 72.2 & -0.9 & +0 & -1.3 & +0 \\
\hline & & \multicolumn{2}{|l|}{ Int } & \multicolumn{2}{|l|}{ Hayes } & 4 & 3 & 74.1 & -14.1 & -14.1 & -14.1 & +0 & 78.5 & -14 & -14.1 & -14.1 & +0 \\
\hline & & \multicolumn{2}{|l|}{ Int } & \multicolumn{2}{|c|}{ Supreme } & 7 & 2 & 99.3 & -14.5 & -17.1 & -14.5 & +0 & 99.4 & +0 & +0 & +0 & +0 \\
\hline & & \multicolumn{2}{|l|}{ Int, String } & \multicolumn{2}{|l|}{ Flares } & 10 & 2 & 90.8 & +0 & +0 & +0 & +0 & 89.2 & +0.3 & +0.3 & +0.3 & +0 \\
\hline & & Int, String & & Kropt & & 6 & 18 & 39.4 & -6.9 & -6.9 & -6.9 & +0 & 68.8 & -3.4 & -3.4 & -3.4 & +0 \\
\hline & & Int, String & & Boxing & & 3 & 2 & 80.7 & -24.4 & -25.2 & -25.2 & -34.1 & 78.5 & -17 & -11.9 & -11.9 & -28.9 \\
\hline & & Int, String & & Flags & & 28 & 2 & 68.2 & -6.2 & -3.6 & -6.7 & $-4.1 *$ & 75.9 & -1 & -2.6 & -2.6 & $-3.1 *$ \\
\hline $\mathrm{NHI}+\mathrm{CA}$ & & Int,Float,Stri & & Diggle & & 8 & 2 & 99.9 & +0 & +0 & +0 & -5.8 & 99.9 & +0 & +0 & +0 & +0 \\
\hline T & & Int, Float & & Hearts & & 13 & 2 & 84.9 & -0.7 & -1.6 & -0.7 & +0 & 86.2 & -1.3 & -3 & -1.3 & +0 \\
\hline & & Int, Float & & Sleuth & & 10 & 2 & 68.9 & -3.3 & -3.3 & -3.3 & +0 & 76.7 & +0 & +0 & +0 & +0 \\
\hline $\mathrm{CA}+\mathrm{NG}$ & & Int, String & & Apnea & & 3 & 2 & 92 & -6.7 & -0.6 & -0.6 & -0.6 & 90.1 & -2.3 & -0.8 & -0.8 & -0.8 \\
\hline $\mathrm{NU}+\mathrm{CA}+\mathrm{ST}$ & & Int, String & & Auto- $\Lambda$ & & 8 & 3 & 89.1 & -4.8 & -8.6 & -8.6 & -15.9 & 95.2 & +0.5 & -18.9 & -18.9 & -20.5 \\
\hline $\mathrm{NU}+\mathrm{CA}+\mathrm{En}$ & & Int,Float,Stri & & Churn & & 19 & 2 & 79.1 & -0.7 & +0.1 & -0.1 & +0.2 & 78.7 & -0.2 & -0.9 & -0.8 & -0.3 \\
\hline $\mathrm{NU}+\mathrm{DT}+\mathrm{EN}$ & & $\begin{array}{l}\text { Int, Float, } \\
\text { String, Date }\end{array}$ & & NYC & & 6 & 15 & 55.8 & +0 & -0.1 & -0.3 & -0.3 & 67.6 & +0 & +0.5 & +0.8 & +0.8 \\
\hline ST & & String & & $\mathrm{BBC}$ & & 1 & 5 & 97.1 & -6.9 & +0 & +0 & +0 & 96.3 & -13. & +0 & +0 & +0 \\
\hline $\mathrm{DT}+\mathrm{ST}$ & & String, Date & & Article & & 3 & 2 & 98.8 & -2.1 & +0 & +0 & +0 & 99.0 & -3.2 & +0 & +0 & +0 \\
\hline $\mathrm{NU}+\mathrm{CA}+\mathrm{ST}+\mathrm{I}$ & & Int,String,PK & & Clothir & & 10 & 5 & 66.7 & -9.2 & -9.1 & -9.2 & +0 & 64.2 & -2.2 & -4.9 & -2.6 & +0 \\
\hline $\mathrm{NU}+\mathrm{DT}+\mathrm{N}$ & & $\begin{array}{l}\text { Int, String, } \\
\text { Time, PK }\end{array}$ & & IOT & & 4 & 2 & 83.8 & -0.3 & +0 & +0 & $+3.6 *$ & 93.8 & -1.4 & +0 & +0 & $+0^{*}$ \\
\hline $\mathrm{NG}+\mathrm{CA}$ & & Int,String, $\mathrm{Pl}$ & & Zoo & & 17 & 5 & 75.6 & -13.4 & -11.1 & -8.9 & -2.2 & 77.8 & -15. & -8.9 & -6.7 & -4.4 \\
\hline $\mathrm{NU}+\mathrm{CA}+\mathrm{EN}+$ & NG & Int,Float,Stri & & PBCse & & 18 & 2 & 68.6 & -1.3 & +0.5 & +0.5 & $+6.2 *$ & 73 & -1.2 & -0.1 & -0.1 & $+2.2^{*}$ \\
\hline $\begin{array}{l}\mathrm{NU}+\mathrm{CA}+\mathrm{LS} \\
+\mathrm{NG}+\mathrm{CS}\end{array}$ & & $\begin{array}{l}\text { Int, Float, } \\
\text { String, PK }\end{array}$ & & Pokem & & 40 & 36 & 65.84 & -52.2 & -52.4 & -52.6 & -0.6 & 88.1 & -3.9 & -3.2 & +0 & +0 \\
\hline $\begin{array}{l}\mathrm{NU}+\mathrm{CA}+\mathrm{D} \\
\mathrm{URL}+\mathrm{NG}+\mathrm{C}\end{array}$ & & $\begin{array}{l}\text { Int,Float, Dat } \\
\text { String, Time }\end{array}$ & & Presid & & 26 & 57 & 39.5 & -7.9 & -7.9 & -8 & -0.9 & 81.7 & -29 & -23.1 & -28.8 & -2.1 \\
\hline (B) Feature & & aw Attribute & & tacet & $|A|$ & & Line & Regress & on $-\mathrm{L} 2 \mathrm{R}$ & egulariza & tion & & & Rand & lom Forest & & \\
\hline (D) Types & & typus & & thet & $\mid$ & & ruth & PD & TFDV & AGL & OurRF & Truth & $P D$ & & TFDV & $\mathrm{AGL}$ & OurRF \\
\hline CA & Int & & $\mathrm{MB}$ & & 2 & & .363 & +0.05 & +0.05 & +0.05 & -0 & 0.384 & $+0 . c$ & & +0.08 & +0.09 & -0 \\
\hline AUI $C A$ & Int & & Vine & eyard & 3 & & 2.97 & +2 & +2 & +2 & -0 & 2.7 & +0.3 & & +0.37 & +0.37 & -0 \\
\hline A & & String & Apn & & 3 & & 206.2 & +62.5 & -0 & -0 & -0 & 1355.7 & +197 & 2.7 & -0 & -0 & -0 \\
\hline DT & Dat & te & Acci & ident & 1 & & 466 & -0 & +384.6 & -0 & -0 & 589.7 & -0 & & +474.8 & -0 & -0 \\
\hline $\begin{array}{l}\mathrm{NU}+\mathrm{CA}+ \\
\mathrm{EN}+\mathrm{NG}\end{array}$ & & , String & Car & Fuel & 11 & & 11.3 & -0.09 & +0.16 & +0.14 & $+0.01 *$ & 11.7 & +0.3 & & +1.1 & +0.9 & $+0.03 *$ \\
\hline
\end{tabular}


over another. Overall, we have 566 columns across 30 downstream datasets. We manually label all the columns with their true feature type. The datasets and their source details are available on the Github repository [25].

\subsection{Models and Metrics}

In terms of downstream model evaluation, we present both extremes of bias-variance tradeoff [29]: L2-regularized Logistic regression (high bias, low variance) for classification, L2-regularized Linear regression (high bias, low variance) for regression, and Random Forest (low bias, high variance) for both classification and regression. Thus, we have 60 downstream models in total. We use the accuracy metric scaled to 100 for the classification tasks and the root mean squared error (RMSE) metric for the regression tasks.

\subsection{Tools compared}

We compare Pandas (PD), TFDV, AutoGluon (AGL), and OurRF, relative to the truth on 30 downstream datasets. We map the feature types inferred by these tools to our label vocabulary as per Figure 3 . Columns that are inferred Numeric are retained as is, Categorical columns are one-hot encoded, Sentence columns are routed through TF-IDF [30], URLs are specially processed through a word-level bigrams, Not-Generalizable columns are dropped, and the rest of the types are featurized with bigrams. After featurization, we use the same methodology as in Section 4.1 for evaluation. Note that one can plug-in any alternate featurization scheme to derive more useful features. However, such feature engineering decisions can be application-specific and are not the focus of this work.

\subsection{Results}

5.4.1 Type Inference Results. Table 4 (A) shows the type inference accuracy of all tools on the downstream datasets. We see that OurRF can correctly infer the feature types for 516 out of 566 columns in these 30 datasets. Pandas has a seemingly high accuracy of $90 \%$ but note the low coverage of columns by its vocabulary, which makes it benefit from high recall. It cannot predict on the other columns at all. The accuracy of TFDV and AutoGluon is much lower than OurRF; their coverage is also slightly lower than OurRF.

5.4.2 Downstream Model Performance. Table 5 presents the end-to-end comparison of downstream models built with feature types inferred by Pandas, AutoGluon, TFDV, and OurRF relative to the true feature types. Table 4 (B) offers summary statistics on how the tools perform relative to the ground truth and other tools. We find that, for a given dataset and a downstream model, OurRF performs worse than the best performing tool for only 13 out of 60 downstream models. Moreover, OurRF underperforms the truth (perfect feature type predictions) for only 20 downstream models. In contrast, Pandas, TFDV, and AutoGluon underperform for significantly more models: 44,35 , and 35 respectively. We present the CDFs of the magnitude of the difference in downstream performance with different approaches compared to Truth in the technical report [17]. We explain the results in-depth below.

1. Why does wrong type inference hurt downstream accuracy? Table 5 shows that wrong type inference almost always leads to a drop in accuracy compared to the accuracy with true feature types.
Moreover, the amount of drop depends upon how many feature types are wrongly classified and how predictive those features are for the target. For instance, wrong type inference leads AutoGluon and TFDV to underperform on 35 out of 60 downstream models. This led to a reduction of an average of $7 \%$ and up to $52 \%$ in accuracy compared to the ground truth-based model. We explain the common patterns of how wrong type inference affected downstream accuracy in the technical report [17].

We empirically studied if the gap in downstream model accuracy caused by wrong type inference relative to the true feature types be bridged by giving multiple representations of the column at the same time to the downstream model. We studied this in the context of Numeric vs Categorical dichotomy of the integer columns of our downstream datasets. We again found that accurate inference of feature types is critical to building accurate downstream models. We present the complete evaluation and additional insights in the technical report [17].

2. Why does wrong type inference of integer Categorical often not hurt downstream Random Forest?

Although the categories encoded as integers in Supreme, Flags, Sleuth, and Vineyard are misclassified by Pandas, AutoGluon, and TFDV, the accuracy of Random Forest either does not drop or drops only marginally. This is because the Categorical features in these datasets are either ordinal and/or have binary domain size. Random Forest has zero bias and thus can potentially represent all categories by doing splits on integers. Linear models, which have lower VCdimension, cannot do this. Thus, the linear models often see much higher accuracy with OurRF than prior tools.

3. How can OurRF exploit user intervention to lift accuracy? Car Fuel has two Embedded Number columns. Although they are predicted correctly by OurRF, a human can intervene to extract their values to use them as Numeric instead of the current bigramization. Thus, a user-in-the-loop can further improve downstream model. Moreover, such intervention can even help Flags where a Categorical feature was erroneously predicted as Context-Specific by OurRF.

4. Why is outperforming truth not necessarily beneficial? On IOT, we observe the lift in accuracy due to a Numeric column called "temp" (denoting temperature) being classified as ContextSpecific. This may not be desired because interpretability can be a concern in this application. Predictions are more explainable when using temperature data as Numeric feature than bigrams. We present more such cases in the technical report [17].

\subsection{Summary}

Overall, OurRF achieves a high accuracy of $91.2 \%$ for inferring feature types on 30 unseen datasets from Kaggle, UCI ML repository, and OpenML. Moreover, we find that wrong feature type inference almost always leads to an accuracy drop for the downstream model relative to the ground truth, except for the Random Forest on ordinal and/or binary domain Categorical. More importantly, our labeled dataset is valuable to build an accurate downstream model because even standard ML models like Random Forest trained on our labeled data achieve the highest accuracy against existing tools for 47 out of 60 downstream models. 


\section{DISCUSSION}

\subsection{Public Release and Leaderboard}

We have released a public repository on GitHub with our entire labeled data for the ML feature type inference task [25]. We also release the pre-trained ML models: $\mathrm{k}-\mathrm{NN}$, logistic regression, RBFSVM, Random Forest, and the CNN. The repository tabulates the precision, recall, and accuracy of all models and existing opensource approaches. The repository includes a leaderboard for public competition on the hosted dataset with 9-class classification accuracy and per-class precision, recall, and binarization accuracy being the metric. We release the downstream benchmark suite containing 30 datasets and the associated code for running the benchmark. Also, we release the raw $1240 \mathrm{CSV}$ files and we invite researchers and practitioners to use our datasets and contribute to augmenting them and creating better featurizations and models.

\subsection{Takeaways}

6.2.1 For Practitioners. We make all the models and featurization routines available for use by wrapping them under functions in a Python library [25]. The ML models can be integrated for feature type inference into existing data prep environments. We have already integrated our pre-trained models with TFDV to improve its inference of Categorical [14]. We are also planning to collaborate with AWS and OpenML on more such integration. We welcome inquiries from more practitioners interested in adopting or enhancing our benchmark. For visual tools such as Excel and Trifacta [28], designing new user-in-the-loop interfaces that account for both model's prediction and human's judgement remains an open research question.

6.2.2 For Researchers. We see three main avenues of improvement for researchers wanting to improve accuracy: better features, better models, and/or getting more labeled data.

First, designing features that can perfectly capture human-level reasoning is an open research question. We found that descriptive stats and attribute names are most useful for prediction, while raw attribute values have only marginal utility. Thus, one can consider designing better featurization routines for them. Second, capturing more semantic knowledge of attributes with an alternative neural architecture is another open problem. Finally, based on our analysis in Section 4.4, one potential way to increase the accuracy is to create more labeled data in categories of examples where ML models get confused, e.g., for List type. Weak supervision and denoising with Snorkel [31] and/or Snuba [32] is one potential mechanism to amplify labeled datasets and teach the ML models to learn better.

\section{RELATED WORK}

AutoML Platforms. Several AutoML tools such as AutoWeka [33] and Auto-sklearn [34] have an automated search process for model selection, allowing users to spend no effort for algorithm selection or hyper-parameter search. However, these AutoML systems do not automate the ML feature type inference task. Several tools perform automatic data transformation steps and generate a set of useful features given a dataset $[35,36]$. However, Deep Feature Synthesis algorithm [36] assumes that the ML feature types are provided explicitly as input, while ExploreKit [35] operates on the syntactic types. Thus, such automatic feature engineering tools can benefit by leveraging the ML models trained on our labeled data.

Other end-to-end AutoML platforms such as Einstein AutoML [3], AutoML Tables [26], and AutoGluon [9] do automate the type inference task. We believe that the standardization of the task and our benchmark labeled dataset is valuable to objectively compare and improve their AutoML platforms. The ML models trained on our labeled dataset can be integrated into such AutoML platforms to improve their type inference accuracy. In addition, other ML platforms such as Airbnb's Zipline [37], Uber's Michelangelo [38], Facebook's FBLearner Flow [39], and commercial platforms such as H20.AI [40] and DataRobot [27] are complementary to our focus and they can also benefit by adopting models trained on our data.

ML Data Prep and Cleaning. Auto-Type [13] is a semantic type detection tool that synthesizes type detection logic for semantic types such as EANCode, Swift Code, etc. But it too is complementary and not directly usable for AutoML just like Sherlock. DataLinter is a rule-based tool that inspects a data file and raises potential data quality issues as warnings to the user [41]. However, ML feature type inference must be done manually. Many works study program synthesis-based approaches [5, 42-44] and/or visual interfaces [28] to reduce manual data transformation grunt work in data prep. There is also much work on reducing data validation and cleaning effort (e.g., [6, 45, 46]). Our work further this general direction on reducing manual effort but it is complementary to all these prior works: our paper is the first to formalize and benchmark ML feature type inference in AutoML platforms.

Database Schema Inference. DB schema inference has been explored in some prior work. Google's BigQuery does syntactic schema detection when loading data from external data warehouses [47]. [48] infers a schema from JSON datasets by performing map and reduce operations using pre-defined rules. But DB schema inference task is syntactic. For instance, the attribute type with integer values has to be identified as an integer. In contrast, with ML type inference the attributes with type integer can be Categorical.

Benchmarks. OpenML AutoML Benchmark focuses on understanding the automation of model selection and hyper-parameter search components of the ML workflow [49]. However, they do not cover any data prep steps. CleanML benchmark focuses on studying the effect of data cleaning operations on downstream models [50]. However, they do not handle the feature type inference task. Thus, both benchmarks are orthogonal to our work.

Data/Model Repositories. OpenML [15] is an open-source collaborative repository for ML practitioners and researchers to share their models, datasets, and workflows for reuse and discussion. Our labeled datasets can be made available to the OpenML community to invite more contributions for augmenting the current labeled dataset and for building more sophisticated models. Hence, our work is complementary to OpenML.

Acknowledgments. This work is supported in part by the National Science Foundation Convergence Accelerator grant under award number OIA-2040727 and gifts from Google and Amazon. We thank the members of UC San Diego's Database Lab, Alkis Polyzotis and Google TFDV team, Julian McAuley, Andreas Müller, Lawrence Saul, and Jingbo Shang for their feedback on this work. 


\section{REFERENCES}

[1] Frank Hutter, Lars Kotthoff, and Joaquin Vanschoren, editors. Automated Machine Learning - Methods, Systems, Challenges. The Springer Series on Challenges in Machine Learning. Springer, 2019.

[2] Google Cloud AutoML, https://cloud.google.com/automl/, Accessed March 22, 2021.

[3] Salesforce Einstein AutoML, https://www.salesforce.com/video/1776007, Accessed March 22, 2021

[4] Pieter Gijsbers, Erin LeDell, Janek Thomas, Sébastien Poirier, Bernd Bischl, and Joaquin Vanschoren. An Open Source AutoML Benchmark. arXiv preprint arXiv:1907.00909, 2019

[5] Zhongjun Jin, Michael R Anderson, Michael Cafarella, and Hosagrahar V Jagadish Foofah: A Programming-By-Example System for Synthesizing Data Transformation Programs. In Proceedings of the 2017 ACM International Conference on Management of Data, pages 1607-1610, 2017.

[6] Sanjay Krishnan, Michael J. Franklin, Ken Goldberg, and Eugene Wu. BoostClean: Automated Error Detection and Repair for Machine Learning. CoRR, abs/1711.01299, 2017.

[7] TransmogrifAI: Automated Machine Learning for Structured Data, https:// transmogrif.ai/, Accessed March 22, 2021.

[8] Denis Baylor, Eric Breck, Heng-Tze Cheng, Noah Fiedel, Chuan Yu Foo, Zakaria Haque, Salem Haykal, Mustafa Ispir, Vihan Jain, Levent Koc, Chiu Yuen Koo, Lukasz Lew, Clemens Mewald, Akshay Naresh Modi, Neoklis Polyzotis, Sukriti Ramesh, Sudip Roy, Steven Euijong Whang, Martin Wicke, Jarek Wilkiewicz, Xin Zhang, and Martin Zinkevich. TFX: A TensorFlow-Based Production-Scale Machine Learning Platform. In Proceedings of the 23rd ACM SIGKDD International Conference on Knowledge Discovery and Data Mining, Halifax, NS, Canada, August 13 - 17, 2017, pages 1387-1395. ACM, 2017.

[9] Nick Erickson, Jonas Mueller, Alexander Shirkov, Hang Zhang, Pedro Larroy, $\mathrm{Mu} \mathrm{Li}$, and Alexander J. Smola. AutoGluon-Tabular: Robust and Accurate AutoML for Structured Data. CoRR, abs/2003.06505, 2020

[10] Olga Russakovsky, Jia Deng, Hao Su, Jonathan Krause, Sanjeev Satheesh, Sean Ma, Zhiheng Huang, Andrej Karpathy, Aditya Khosla, Michael S. Bernstein, Alexander C. Berg, and Fei-Fei Li. ImageNet Large Scale Visual Recognition Challenge. Int. F. Comput. Vis., 115(3):211-252, 2015.

[11] Wes McKinney. pandas: a Foundational Python Library for Data Analysis and Statistics. Python for High Performance and Scientific Computing, 14, 2011.

[12] Madelon Hulsebos, Kevin Hu, Michiel Bakker, Emanuel Zgraggen, Arvind Satyanarayan, Tim Kraska, Çagatay Demiralp, and César Hidalgo. Sherlock: A Deep Learning Approach to Semantic Data Type Detection. In Proceedings of the 25th ACM SIGKDD International Conference on Knowledge Discovery \& Data Mining, pages $1500-1508,2019$.

[13] Cong Yan and Yeye He. Synthesizing Type-Detection Logic for Rich Semantic Data Types Using Open-Source Code. In Proceedings of the 2018 International Conference on Management of Data, pages 35-50, 2018.

[14] Vraj Shah, Kevin Yang, and Arun Kumar. Improving Feature Type Inference Accuracy of TFDV with SortingHat, Accessed March 22, 2021. https: //adalabucsd.github.io/papers/TR_2020_TFDV.pdf.

[15] Joaquin Vanschoren, Jan N. van Rijn, Bernd Bischl, and Luís Torgo. OpenML networked science in machine learning. SIGKDD Explor., 15(2):49-60, 2013

[16] URL Standards, https://url.spec.whatwg.org, Accessed March 22, 2021

[17] Vraj Shah, Jonathan Lacanlale, Premanand Kumar, Kevin Yang, and Arun Kumar. Towards Benchmarking Feature Type Inference for AutoML Platforms, Accessed March 22, 2021. https://adalabucsd.github.io/papers/TR_2021_SortingHat.pdf.

[18] Christopher Ré, Feng Niu, Pallavi Gudipati, and Charles Srisuwananukorn. Overton: A Data System for Monitoring and Improving Machine-Learned Products. arXiv preprint arXiv:1909.05372, 2019.

[19] Vincent Chen, Sen Wu, Alexander J Ratner, Jen Weng, and Christopher Ré. Slicebased Learning: A Programming Model for Residual Learning in Critical Data Slices. In Advances in neural information processing systems, pages 9397-9407, 2019.

[20] Yeounoh Chung, Tim Kraska, Neoklis Polyzotis, Ki Hyun Tae, and Steven Euijong Whang. Slice Finder: Automated Data Slicing for Model Validation. In 2019 IEEE 35th International Conference on Data Engineering (ICDE), pages 1550-1553. IEEE, 2019.

[21] Ki Hyun Tae and Steven Euijong Whang. Slice Tuner: A Selective Data Collection Framework for Accurate and Fair Machine Learning Models. arXiv preprint arXiv:2003.04549, 2020

[22] Xiang Zhang, Junbo Jake Zhao, and Yann LeCun. Character-level Convolutional Networks for Text Classification. In Corinna Cortes, Neil D. Lawrence, Daniel D. Lee, Masashi Sugiyama, and Roman Garnett, editors, Advances in Neural Information Processing Systems 28: Annual Conference on Neural Information Processing Systems 2015, December 7-12, 2015, Montreal, Quebec, Canada, pages 649-657, 2015.

[23] Xiang Zhang and Yann LeCun. Text Understanding from Scratch. CoRR, abs/1502.01710, 2015.

[24] Dmitry Duplyakin, Robert Ricci, Aleksander Maricq, Gary Wong, Jonathon Duerig, Eric Eide, Leigh Stoller, Mike Hibler, David Johnson, Kirk Webb, Aditya
Akella, Kuangching Wang, Glenn Ricart, Larry Landweber, Chip Elliott, Michael Zink, Emmanuel Cecchet, Snigdhaswin Kar, and Prabodh Mishra. The Design and Operation of CloudLab. In Proceedings of the USENIX Annual Technical Conference (ATC), pages 1-14, July 2019.

[25] Github Repository for ML Feature Type Inference, https://github.com/pvn25/MLData-Prep-Zoo/tree/master/MLFeatureTypeInference, Accessed March 22, 2021.

[26] Google AutoML Tables, https://cloud.google.com/automl-tables, Accessed March 22, 2021.

[27] DataRobot, https://www.datarobot.com, Accessed March 22, 2021.

[28] Trifacta: Data Wrangling Tools \& Software, https://www.trifacta.com/, Accessed March 22, 2021.

[29] Trevor Hastie, Jerome H. Friedman, and Robert Tibshirani. The Elements of Statistical Learning: Data Mining, Inference, and Prediction. Springer Series in Statistics. Springer, 2001.

[30] Juan Ramos. Using TF-IDF to Determine Word Relevance in Document Oueries. In Proceedings of the first instructional conference on machine learning, volume 242, pages 133-142. New Jersey, USA, 2003.

[31] Alexander Ratner, Stephen H Bach, Henry Ehrenberg, Jason Fries, Sen Wu, and Christopher Ré. Snorkel: Rapid Training Data Creation with Weak Supervision. Proceedings of the VLDB Endowment, 11(3):269-282, 2017.

[32] Paroma Varma and Christopher Ré. Snuba: Automating Weak Supervision to Label Training Data. In Proceedings of the VLDB Endowment. International Conference on Very Large Data Bases, volume 12, page 223. NIH Public Access, 2018.

[33] Chris Thornton, Frank Hutter, Holger H Hoos, and Kevin Leyton-Brown. AutoWEKA: Combined Selection and Hyperparameter Optimization of Classification Algorithms. In Proceedings of the 19th ACM SIGKDD international conference on Knowledge discovery and data mining, pages 847-855. ACM, 2013.

[34] Matthias Feurer, Aaron Klein, Katharina Eggensperger, Jost Tobias Springenberg, Manuel Blum, and Frank Hutter. Efficient and Robust Automated Machine Learning. In Corinna Cortes, Neil D. Lawrence, Daniel D. Lee, Masashi Sugiyama, and Roman Garnett, editors, Advances in Neural Information Processing Systems 28: Annual Conference on Neural Information Processing Systems 2015, December 7-12, 2015, Montreal, Quebec, Canada, pages 2962-2970, 2015.

[35] Gilad Katz, Eui Chul Richard Shin, and Dawn Song. ExploreKit: Automatic Feature Generation and Selection. In 2016 IEEE 16th International Conference on Data Mining (ICDM), pages 979-984. IEEE, 2016.

[36] James Max Kanter and Kalyan Veeramachaneni. Deep feature synthesis: Towards automating data science endeavors. In 2015 IEEE international conference on data science and advanced analytics (DSAA), pages 1-10. IEEE, 2015.

[37] Airbnb Zipline, https://conferences.oreilly.com/strata/strata-ny-2018/public/ schedule/detail/68114, Accessed March 22, 2021.

[38] Uber Michelangelo, https://eng.uber.com/michelangelo/, Accessed March 22, 2021.

[39] Facebook's FBLearner Flow, https://engineering.fb.com/core-data/introducingfblearner-flow-facebook-s-ai-backbone/, Accessed March 22, 2021

[40] H2o.AI, https://www.h2o.ai/, Accessed March 22, 2021.

[41] Nick Hynes, D Sculley, and Michael Terry. The Data Linter: Lightweight, Automated Sanity Checking for ML Data Sets. In NIPS MLSys Workshop, 2017.

[42] Sumit Gulwani. Automating String Processing in Spreadsheets Using InputOutput Examples. In ACM Sigplan Notices, volume 46, pages 317-330. ACM, 2011.

[43] Sumit Gulwani, William R. Harris, and Rishabh Singh. Spreadsheet data manipulation using examples. Commun. ACM, 55(8):97-105, 2012.

[44] Yeye $\mathrm{He}, \mathrm{Xu} \mathrm{Chu}$, Kris Ganjam, Yudian Zheng, Vivek Narasayya, and Surajit Chaudhuri. Transform-Data-by-Example (TDE): An Extensible Search Engine for Data Transformations. Proceedings of the VLDB Endowment, 11(10):1165-1177, 2018.

[45] Sanjay Krishnan, Michael J. Franklin, Ken Goldberg, Jiannan Wang, and Eugene Wu. ActiveClean: An Interactive Data Cleaning Framework For Modern Machine Learning. In Fatma Özcan, Georgia Koutrika, and Sam Madden, editors, Proceedings of the 2016 International Conference on Management of Data, SIGMOD Conference 2016, San Francisco, CA, USA, June 26 - July 01, 2016, pages 2117-2120. ACM, 2016.

[46] Sebastian Schelter, Dustin Lange, Philipp Schmidt, Meltem Celikel, Felix Biessmann, and Andreas Grafberger. Automating Large-Scale Data Quality Verification. Proceedings of the VLDB Endowment, 11(12):1781-1794, 2018.

[47] Schema Detection BigQuery, https://cloud.google.com/bigquery/docs/schemadetect, Accessed March 22, 2021.

[48] Mohamed-Amine Baazizi, Houssem Ben Lahmar, Dario Colazzo, Giorgio Ghelli, and Carlo Sartiani. Schema Inference for Massive JSON Datasets. In Extending Database Technology (EDBT), 2017.

[49] Pieter Gijsbers, Erin LeDell, Janek Thomas, Sébastien Poirier, Bernd Bischl, and Joaquin Vanschoren. An Open Source AutoML Benchmark. arXiv preprint arXiv:1907.00909, 2019.

[50] Peng Li, Xi Rao, Jennifer Blase, Yue Zhang, Xu Chu, and Ce Zhang. CleanML: A Benchmark for Joint Data Cleaning and Machine Learning [Experiments and Analysis]. arXiv preprint arXiv:1904.09483, 2019. 